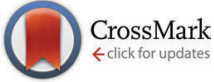

Cite this: J. Mater. Chem. C, 2017, 5, 1668

Received 15th December 2016, Accepted 8th January 2017

DOI: $10.1039 / c 6 t c 05413 g$

rsc.li/materials-c

\section{Colloidal dispersions of conducting copolymers of aniline and $p$-phenylenediamine for films with enhanced conductometric sensitivity to temperature}

\begin{abstract}
Yu Li, ${ }^{\text {ab }}$ Patrycja Bober, ${ }^{\star a}$ Miroslava Trchováa and Jaroslav Stejskal ${ }^{a}$
Polyaniline colloids stabilized with poly( $N$-vinylpyrrolidone) were prepared by the oxidation of aniline with peroxydisulfate. The replacement of a part of aniline with $p$-phenylenediamine converted the original globular colloidal particles into nanofibers, which is a favorable morphology for the deposition of conducting patterns, but the colloidal stability was retained. The corresponding poly(aniline-co- $p$ phenylenediamine) powders have been characterized using FTIR spectroscopy. The copolymers have a more pronounced temperature dependence compared with the parent polyaniline; the ratio of the conductivity at 300 and $100 \mathrm{~K}$ is 24 for polyaniline, 235 for a copolymer containing 20 mol\% p-phenylenediamine, and 3690 for 30 mol\% p-phenylenediamine. Due to their fibrillar morphology, copolymer colloids prepared with 10 and 20 mol\% p-PDA produced films with conductivity nearly three orders of magnitude higher than that from globular PANI colloids. The conductivity of the film cast from the copolymer colloid prepared with 20 mol\% p-PDA exhibits a temperature dependence much steeper than its powder counterpart. The processable copolymer colloids are thus well suited for the preparation of conducting temperature-sensitive films.
\end{abstract}

\section{Introduction}

Poly( $p$-phenylenediamine $)^{1,2}$ and copolymers of aniline with $p$-phenylenediamine ( $p$-PDA) are of special interest as derivatives in the polyaniline family of conducting polymers, because of their unique electrical properties and morphological characteristics. The homopolymer of aniline, polyaniline, exhibits a good conductivity up to $1-10 \mathrm{~S} \mathrm{~cm}^{-1}$, while poly $(p$-phenylenediamine) (PPDA) is virtually insulating with conductivity below $10^{-9} \mathrm{~S} \mathrm{~cm}^{-1}$. The copolymerization of aniline and $p$-PDA, however, yields products, which show a conductivity dependent on the ratio of aniline or $p$-PDA in the monomer mixture. ${ }^{4}$ In particular when a certain amount of $p$-PDA was used, the conductivity of copolymers was even higher than that of pristine polyaniline. ${ }^{5,6}$ It was also found that the conductivity of copolymers of aniline and $p$-PDA, poly(aniline-co-p-PDA), containing a medium content of $p$-PDA had a much stronger dependence on temperature than either polyaniline or poly( $p$-phenylenediamine) alone. ${ }^{4,7}$ Therefore, by carrying out copolymerization of aniline and $p$-PDA, it is possible to tailor the

\footnotetext{
${ }^{a}$ Institute of Macromolecular Chemistry, Academy of Sciences of the Czech Republic, 16206 Prague 6, Czech Republic. E-mail: bober@imc.cas.cz

${ }^{b}$ Department of Applied Chemistry, School of Science, Xi'an Jiaotong University,

Xi'an 710049, People's Republic of China
}

electrical properties of these kinds of semiconducting materials just by adjusting the mole ratio of the co-monomers. ${ }^{8}$ This is quite attractive for developing flexible temperature sensors. Even more interestingly, pellistors are solid-state devices used to detect combustible gases. ${ }^{9}$ They operate upon catalytic combustion of gases, and heat is produced as a response. The associated increase in temperature can be converted into a conductometric change. The use of temperature-sensitive materials may obviously greatly improve the detection limit of pellistors.

On the other hand, a small amount of $p$-PDA introduced into the aniline polymerization system accelerates the polymerization course, ${ }^{10,11}$ and promotes the formation of one-dimensional (1-D) polyaniline nanofibers with a remarkably increased aspect ratio as well as the yield. For example, it has been widely reported that 1-D polyaniline nanofibers with high aspect ratios were obtained by adding a small amount of $p$-PDA to the reaction mixture. ${ }^{12-15}$ This role of $p$-PDA provides an efficient way to achieve PANI nanostructures with both the one-dimensional morphology and improved conductivity. Moreover, $p$-PDA also possesses reversible redox electrochemical activity and improves the chemical stability of copolymerized PANI. ${ }^{6,16}$ Thus, the incorporation of $p$-PDA into aniline chains through chemical or electrochemical polymerization offers options to obtain electroactive materials with improved properties which are attractive for many application processes. ${ }^{6,17-19}$ 
Nevertheless, copolymers of aniline and $p$-PDA prepared through chemical oxidative polymerization reported in the literature are mainly powders. ${ }^{3,20,21}$ The copolymers in the solid state are poorly soluble in common solvents and impossible to melt before degradation ${ }^{20}$ and, consequently, they are not attractive for applications in small-sized flexible electronic devices. Electrochemical copolymerization of aniline and $p$-PDA can deposit the copolymers on electrodes, ${ }^{19,22}$ however, the specific requirements on the electrolytes and electrode substrate materials restrict their applicability. Therefore, aniline and $p$-PDA copolymers in various forms such as suspensions, ${ }^{23}$ dispersions, ${ }^{18}$ hydrogels ${ }^{14}$ and nanocomposites ${ }^{24-26}$ have been developed in order to make use of their application properties. The dispersion polymerization of aniline in the presence of water-soluble polymers yields conducting-polymer colloids in an aqueous medium, ${ }^{27}$ which can be easily used in film-forming processes such as dip-coating, spin-coating and various printing technologies. ${ }^{28}$ However, considering the conductivity of the film cast from conducting polymer colloidal dispersion, conducting nanoparticles embedded in the matrix of non-conducting stabilizers usually lead to low film conductivity. If the morphology of colloidal particles can be elongated and simultaneously they possess a higher conductivity, a conducting network can easily form in the colloidal film and thus reduce the conducting percolation threshold, providing conductive colloidal films.

To sum up, the present study addresses three tasks: (1) the synthesis of conducting materials with improved temperature sensitivity, (2) their preparation in colloidal forms suitable for film forming processes, (3) with special attention paid to the conversion of the globular polymer morphology into one-dimensional nanofibers that display a lower percolation limit and improve conductivity in the composites.

\section{Experimental}

\section{Synthesis}

Aniline hydrochloride (Penta, Czech Republic), 1,4-phenylenediamine dihydrochloride (Sigma-Aldrich), ammonium peroxydisulfate (APS; Penta, Czech Republic) and poly( $N$-vinylpyrrolidone) (PVP; $M=360000$; Sigma-Aldrich) were used as received. PVP solution (4 wt\%) was prepared by dissolving PVP in Milli-Q water. Monomers at various mole ratios were added to each $5 \mathrm{~mL}$ portion of PVP solution. After the monomers dissolved, $5 \mathrm{~mL}$ of APS solution in Milli-Q water was added and mixed. The starting concentrations of reactants thus were $0.2 \mathrm{M}$ aniline and $p$-phenylenediamine, and $0.25 \mathrm{M}$ APS in $2 \mathrm{wt} \%$ PVP solution. After $24 \mathrm{~h}$ at room temperature, the reaction products were poured into dialysis tube (Visking Dialysis Membrane, supplied by Carl Roth GmbH, Germany, molecular-weight cutoff 14000 ) for exhaustive dialysis against $0.1 \mathrm{M}$ hydrochloric acid for one week. The corresponding copolymer powders were prepared in the same manner but in the absence of PVP. Powders were collected by filtration, rinsed with acetone and dried in air.

\section{Characterization}

The morphology of colloidal particles was examined using a transmission electron microscope (TEM, JEOL JEM200X). The colloidal dispersion was $200 \times$ times diluted with $0.1 \mathrm{M}$ hydrochloric acid and treated in an ultrasonic bath for $15 \mathrm{~min}$ for particle-size evaluation using a dynamic light-scattering apparatus (AutoSizer Lo-C, Malvern, UK) and the UV-visible spectra (Lambda 950 spectrometer, Perkin Elmer, UK) were recorded.

The four-point probe method was employed for determining the conductivity of films cast from copolymer colloids. Polyaniline, PPDA, and copolymer colloids were coated on glass supports $1 \mathrm{~cm}$ in diameter by drop-casting and left to dry overnight. Signatone Corporation manual four-point resistivity probing equipment was used along with a Keithley 2700 for the conductivity measurements. The conductivity of powders compressed to pellets with a manual hydraulic press at $540 \mathrm{MPa}$ was also determined by a four-point method in van der Pauw arrangement with a Keithley 220 Programmable Current Source, a Keithley 2010 Multimeter as a voltmeter, and a Keithley 705 Scanner equipped with a Keithley 7052 Matrix Card.

A Thermo Nicolet NEXUS 870 FTIR Spectrometer (Madison, WI, USA) in the wavenumber range of 400 to $4000 \mathrm{~cm}^{-1}$ has been used for the determination of the infrared spectra. Powdered samples were characterized ex situ in the transmission mode after dispersion in potassium bromide pellets.

\section{Results and discussion}

\section{Copolymer formation}

By referring to the results reported in the literature, we have proposed the mechanism of copolymer formation (Scheme 1). We also noticed that the copolymerization system turned dark blue followed by green color from the initial colourless state almost in a few seconds after the APS solution was mixed with the monomer solution. The yield of copolymers in powder form was high. For instance, $0.81 \mathrm{~g}$ of products per $1 \mathrm{~g}$ of monomer was obtained for the aniline homo-polymerization, and $0.88 \mathrm{~g}$ of products per $1 \mathrm{~g}$ of monomer for the copolymerization with $10 \mathrm{~mol} \%$ p-PDA, i.e. a $9 \%$ increase of the yield, was achieved (Fig. 1). The yield in the copolymerization with $20 \mathrm{~mol} \% p$-PDA, $0.87 \mathrm{~g}$ of products per $\mathrm{g}$ of monomer, was also higher than that of neat PANI.

\section{Conductivity of copolymers}

The introduction of $p$-PDA along with aniline in its chemical polymerization not only produces copolymers with conductivity dependent on the $p$-PDA mole fraction, but also significantly affects the temperature dependence of the conductivity of produced copolymers (Fig. 2). The conductivity of pristine PANI increased mildly with increasing temperature as typical of organic semiconductors. ${ }^{29,30}$ With more $p$-PDA introduced into the copolymerization mixture, the room temperature conductivities of the copolymers decreased nearly linearly on a semilogarithmic scale, indicating that the copolymerization products are statistical copolymers instead of a mixture of the 
<smiles>Nc1ccc(N)cc1</smiles><smiles>Nc1ccccc1C=C[C-]C1C=CC(=[NH2+])C=CC1</smiles>

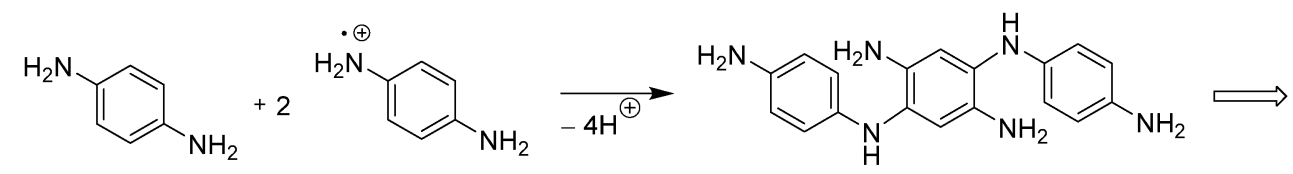<smiles></smiles>

Scheme 1 A possible structure of aniline and $p$-phenylenediamine copolymers.

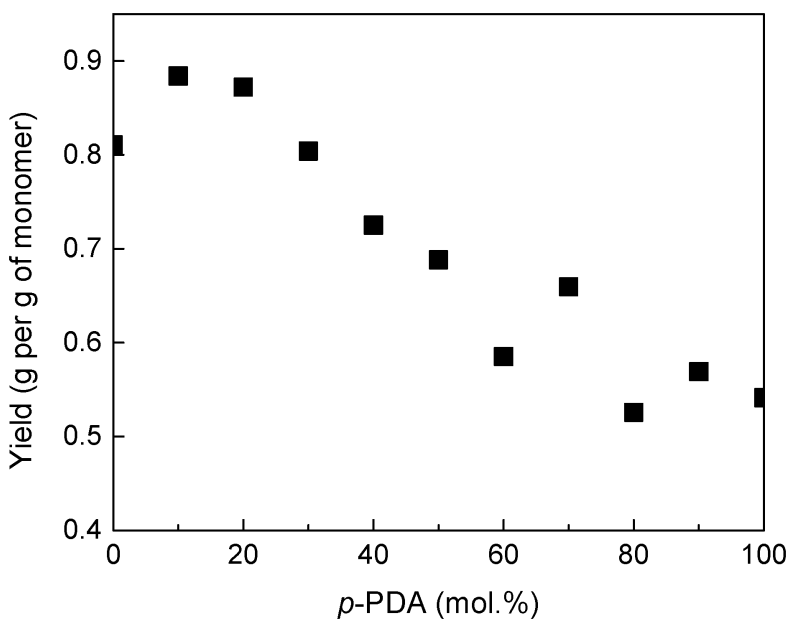

Fig. 1 The yield of aniline and $p$-PDA copolymers with various $p$-PDA mole fractions in the feed.

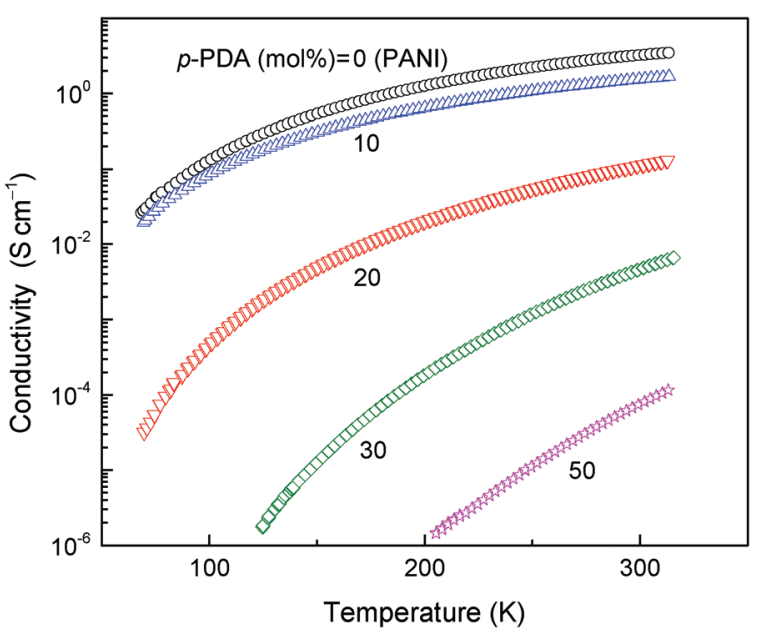

Fig. 2 Temperature dependence of the conductivity of poly(aniline-co$p$-phenylenediamine) with various mole fractions of $p$-PDA. corresponding homopolymers. ${ }^{1,31}$ The reduction in conductivity corresponds to the shift in dependence in Fig. 2 downwards as the content of $p$-PDA increased. Considering the temperature dependence of copolymer conductivity, especially in the lowtemperature region the dependence became stronger (Fig. 2). The ratio of conductivity at $100 \mathrm{~K}$ to that at $300 \mathrm{~K}$ was 24 for polyaniline, and increased to 235 for a copolymer containing $20 \mathrm{~mol} \% p$-PDA, and to 3690 for $30 \mathrm{~mol} \% p$-PDA. It is important to realize that conductivity in Fig. 2 is plotted on a semilogarithmic scale. Copolymers prepared with $20-30 \mathrm{~mol} \%$ p-PDA which have a moderate conductivity over a wide temperature range as well as a conductivity change over several orders of magnitude are promising for producing materials which are able to respond sensitively to the temperature change. For the copolymer prepared with $50 \mathrm{~mol} \% \mathrm{p}$-PDA, the temperature dependence of conductivity is still steep but the level of conductivity at low temperatures becomes too low for practical sensing applications.

The fact that aniline and $p$-PDA copolymers exhibit enhanced conductometric sensitivity to temperature can be explained as follows. $p$-Phenylenediamine disrupts the conducting sequences of the aniline constitutional units. The charge carriers can overcome such defects to some extent at room temperature but not after the temperature was reduced. For that reason, polyaniline sequences control the transport at room temperature, while mainly sequences of $p$-PDA units determine the conductivity at low temperature. Due to a large difference in the conductivities of parent homopolymers, ${ }^{31}$ units of $\mathrm{S} \mathrm{cm}^{-1}$ for polyaniline and $10^{-9} \mathrm{~S} \mathrm{~cm}^{-1}$ for PPDA, the conductivity of copolymers becomes strongly temperature-dependent, compared to both parent homopolymers that display only a moderate dependence of conductivity on temperature. ${ }^{31}$ The conductivity behavior of copolymers can also be discussed at the level of disorder introduced by nonconducting units into the semi-conducting phase. ${ }^{32}$

\section{Infrared spectroscopy}

Infrared spectroscopy was used to assess the molecular structure of oxidation products and to prove that copolymers, and not only a mixture of homopolymers, were obtained. The infrared 
spectrum of pristine PANI changes as $p$-PDA is introduced into the reaction mixture during chemical co-polymerization (Fig. 3). For low content of $p$-PDA (10-20 mol\%) the spectra are very flat due to the stone-like structure of the samples. However, the main bands of PANI with maxima at 1556, 1480, 1300, 1241, 1142 , and $800 \mathrm{~cm}^{-1}$ can be recognized. ${ }^{33}$ When the concentration of $p$-PDA increased to $30-40 \mathrm{~mol} \%$, the bands of the spectrum of PANI still prevail and some weak bands of PPDA can be observed at 1502, 1159 and $827 \mathrm{~cm}^{-1} \cdot{ }^{34}$ With increasing mole fraction of $p$-PDA fed as the monomer, the band at $1572 \mathrm{~cm}^{-1}$ became more obvious, indicating an increasing amount of quinonediimine units compared to that in the ladder-like PPDA. ${ }^{35}$ For $50-60 \mathrm{~mol} \%$ of $p$-PDA the bands of PANI situated at 1300 and $1241 \mathrm{~cm}^{-1}$ characteristic of the conducting form of PANI decreased and the bands of PPDA dominate the spectra. When the concentration of $p$-PDA reached $70-100 \mathrm{~mol} \%$, the spectra of the products became virtually identical to the spectrum of pristine PPDA. ${ }^{36}$

\section{Colloids and their morphology}

In spite of their attractive conductivity performance, the copolymers of aniline and $p$-PDA in the powder form are far away from practical applications especially in the fields such as flexible small-sized electronic devices. In the next step, the preparation of processable colloidal forms was investigated. Copolymerization of aniline and $p$-PDA in the presence of the PVP stabilizer makes it feasible to produce aqueous dispersions of one-dimensional nanostructures at a high monomer concentration. When the aniline polymerization was carried out in the presence of $2 \mathrm{wt} \% \mathrm{PVP}$, well-dispersed nanoparticles with diameters around $200 \mathrm{~nm}$ were obtained, and entangled 1-D nanofibrils were present in the copolymerization system containing $p$-PDA (Fig. 4). As observed in the TEM images, the typical products of aniline and $p$-PDA copolymers from the dispersion system are 1-D nanostructures composed of thin nanofibrils with diameters $\approx 50 \mathrm{~nm}$, which have a much higher aspect ratio than the aniline homo-polymerization products.

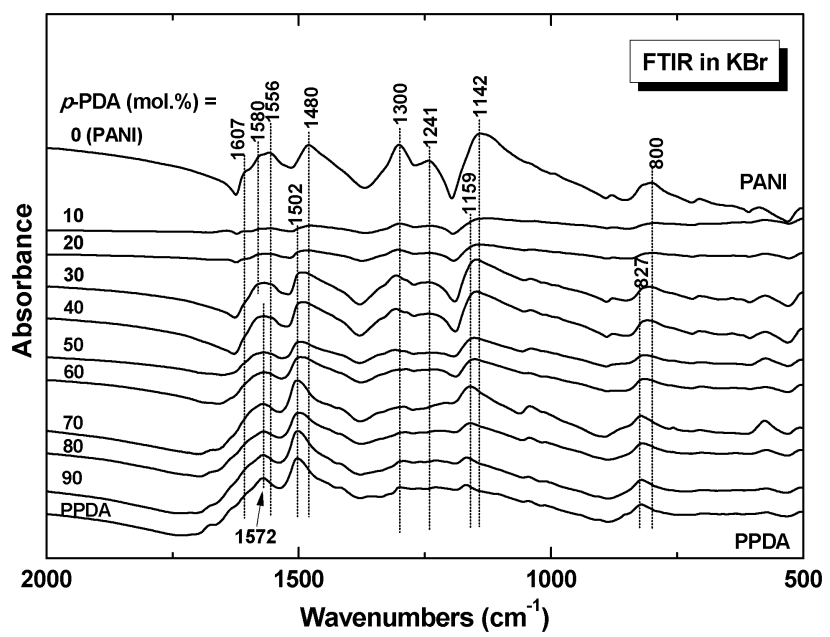

Fig. 3 FTIR spectra of the products of copolymerization of aniline and $p$-PDA with different $p$-PDA feeding mole fractions.
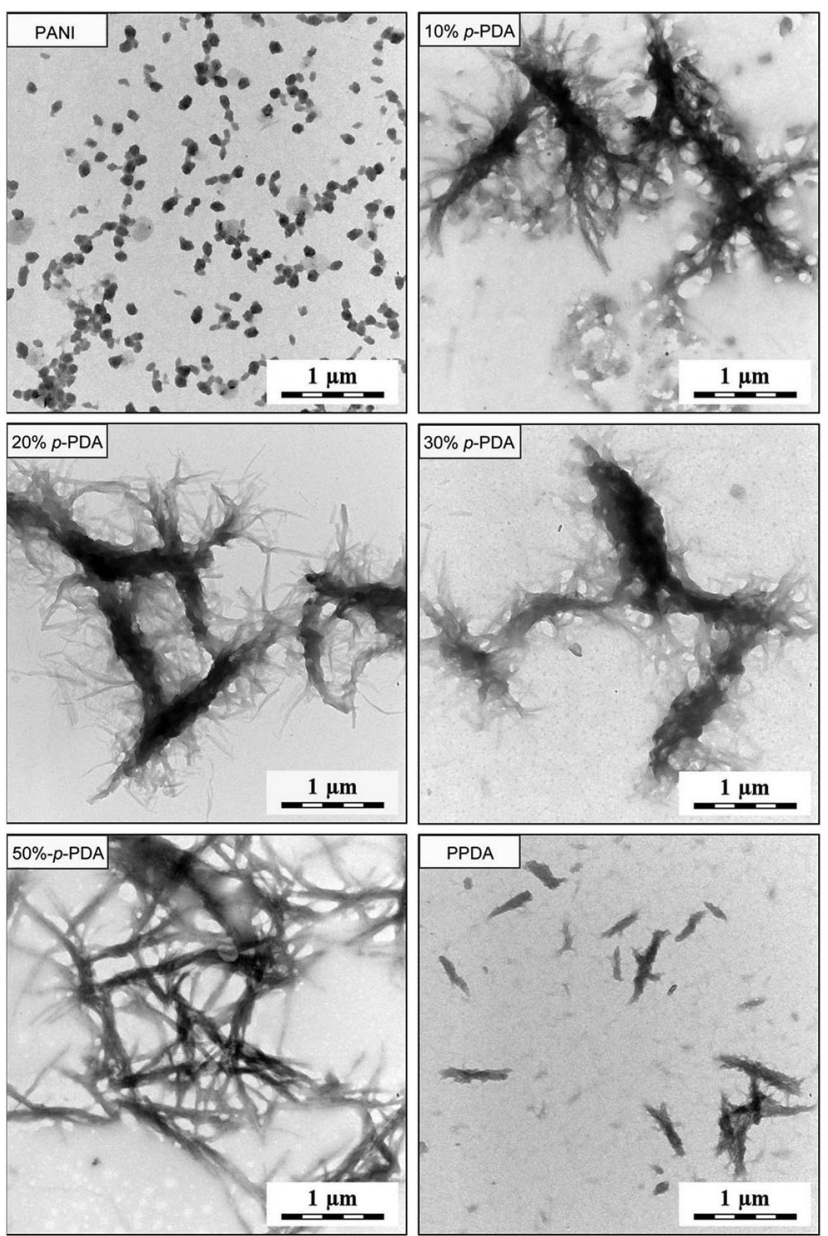

Fig. 4 Morphology of the copolymer colloids prepared with different mole fractions of $p$-PDA.

The formation of 1-D nanostructures in the copolymerization system can be mainly attributed to the role of $p$-PDA, which can be incorporated into the copolymer chains and form phenazinelike or ladder-like planar segments. These planar structures can self-assemble through $\pi-\pi$ stacking into one-dimensional nanostructures that serve as nucleation centers directing the growth of copolymers chains. ${ }^{12,37}$ It is also possible that the accelerated polymerization rate, which favors the homogeneous nucleation of polymerization products, facilitates the 1-D morphology over other irregular aggregates. ${ }^{38}$

\section{Particle size and size distribution}

Dynamic light scattering measurements on the dispersion of colloids showed that the average particle size and dispersity index of the copolymer colloids reached their maximum when the mole percentage of $p$-PDA was about $20 \%$ (Table 1), and decreased with a further increase of the $p$-PDA. When aniline and $p$-PDA were fed at a 1:1 mole ratio to the dispersion polymerization system, the as-prepared product showed the morphology of nanofibers with lengths up to the micrometer scale, while these 1-D structures may be broken during a long period treatment in an ultrasonic bath. ${ }^{39}$ After the ultrasonic 
Table 1 Particle sizes, $D$, and dispersity index, DI, determined before and after dialysis by dynamic light scattering from poly(aniline-co- $p$-phenylenediamine) dispersions prepared at various mole fractions of $p$-phenylenediamine, $x$

\begin{tabular}{lcllll}
\hline & \multicolumn{2}{c}{ Before dialysis } & & \multicolumn{2}{c}{ After dialysis } \\
\cline { 2 - 3 }$x,(\mathrm{~mol} \% p$-PDA $)$ & $D(\mathrm{~nm})$ & DI & & $D(\mathrm{~nm})$ & DI \\
\hline $0(=$ PANI $)$ & 235 & 0.25 & & 248 & 0.24 \\
10 & 848 & 0.81 & & 1134 & 0.80 \\
20 & 1029 & 0.85 & & 1554 & 0.76 \\
30 & 936 & 0.75 & & 783 & 0.75 \\
50 & 628 & 0.93 & & 617 & 0.46 \\
$100(=$ PPDA $)$ & 536 & 0.45 & & 420 & 0.20
\end{tabular}

treatment period was beyond $30 \mathrm{~min}$, the color of the blue colloid dispersion of the copolymer prepared with $50 \mathrm{~mol} \%$ $p$-PDA slowly became lighter and almost colorless. Both the averaged particle sizes of poly(aniline-co-50 mol\% $p$-PDA) and poly( $p$-phenylenediamine) reduced to be around $350 \mathrm{~nm}$ after 30 min of ultrasonic treatment. Though the average particle size determined from the DLS method is different from that obtained from TEM images, the trend of the particle size change with the increasing $p$-PDA feed ratio is generally consistent with the morphological changes observed by TEM. Furthermore, for all the copolymer particles with 1-D morphology, a dispersity index below 1.0 proves their colloidal nature and guarantees the good processability of copolymer dispersions. The dialysis, which removes all low-molecular-weight compounds, such as residual reactants and by-products, is needed before the dispersions could be applied in the preparation of films. This process does not affect the colloidal parameters of the dispersions (Table 1). No aggregation of particles was observed during the storage.

\section{UV-vis spectra}

The colloidal nature makes it convenient to record the UV-visible spectra of the copolymers in aqueous medium, ${ }^{40}$ which clearly showed the influence of $p$-PDA on the conjugation structure of the copolymerization products (Fig. 5). In the UV-vis spectrum of PANI, the band around $400 \mathrm{~nm}$ and another band beyond $750 \mathrm{~nm}$ represent the polaron state which is typical for aciddoped PANI samples. ${ }^{12,41}$ With increasing $p$-PDA fed as the monomer, the copolymerization products showed reduced absorption intensity of the "tail" around $750 \mathrm{~nm}$, whereas an additional band around $300 \mathrm{~nm}$ belonging to the $\pi-\pi^{*}$ transition of benzenoid rings became more evident, indicating that more $p$-PDA units were involved in the polymer chains. ${ }^{10}$ When the mole percentage of $p$-PDA was $50 \%$, i.e. equal mole of $p$-PDA and aniline was used, the UV-vis spectrum of the polymerization products almost totally lost the characteristic of polaron transition, and a broad band appeared in the region of 500-650 nm instead of a tail extending beyond $750 \mathrm{~nm}$, which is close to the spectrum of neat PPDA. ${ }^{34}$ Similarly to FTIR spectra, the gradual transition of the position of the absorption bands in the spectra with increasing $p$-PDA mole ratio further supports the fact that the products are copolymers composed of statistically

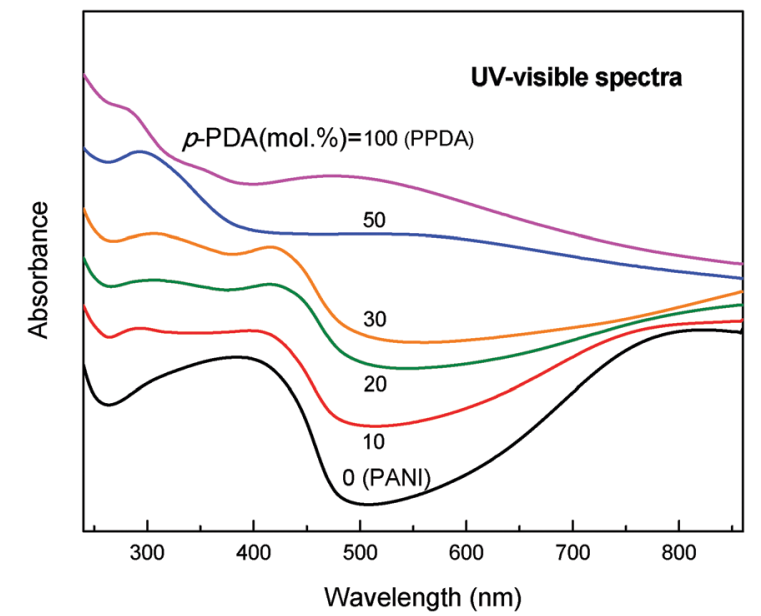

Fig. 5 UV-vis spectra of aniline and p-PDA copolymers with various $p$-PDA feeding mole fractions.

distributed aniline and $p$-PDA units, instead of a simple mixture of PANI and $p$-PDA. ${ }^{13}$

\section{Conductivity of films cast from colloids}

The colloidal dispersions of copolymers are suitable for dropcasting or dip-coating to form continuous uniform conducting or semi-conducting films on substrates such as glass. Despite the fact that the film made of PANI colloids was green, which indicates that the polyaniline was in the protonated conducting state, the film conductivity was rather low (Table 2). This is reasonable as the small-sized PANI colloidal particles (Fig. 4) are evenly dispersed in the insulating matrix of the PVP stabilizer and thus cannot form efficient conducting pathways. Copolymer colloids prepared with 10 and 20 mol\% $p$-PDA produced film conductivity nearly three orders of magnitudes higher than that from neat PANI colloids (Table 2). In spite of the fact that the same copolymers in the powder form exhibit conductivity comparable to or slightly lower that of neat PANI (Fig. 2), the significantly increased film conductivity of the copolymer containing $10 \mathrm{~mol} \% p$-PDA and $20 \mathrm{~mol} \% p$-PDA can be attributed to the highly increased aspect ratio of copolymer particles, which are able to connect to effective conducting pathways. It is no wonder that the copolymer containing $20 \mathrm{~mol} \% \mathrm{p}$-PDA which possesses the largest particle size (Table 1) showed the highest film conductivity.

Furthermore, the film conductivity of the copolymer colloid prepared with $20 \mathrm{~mol} \% \mathrm{p}$-PDA still retains the large temperature dependence as its counterpart in powder form (Fig. 6).

Table 2 Thickness and conductivity of drop-cast films prepared from copolymer colloids

\begin{tabular}{lcl}
\hline$x(\mathrm{~mol} \% p$-PDA $)$ & Thickness $(\mu \mathrm{m})$ & Conductivity $\left(\mathrm{S} \mathrm{cm}^{-1}\right)$ \\
\hline 0 (PANI) & 16.9 & $4.9 \times 10^{-5}$ \\
10 & 7.7 & $2.1 \times 10^{-2}$ \\
20 & 5.5 & $8.4 \times 10^{-2}$ \\
30 & 3.5 & $1.6 \times 10^{-3}$ \\
50 & 11.9 & $2.2 \times 10^{-6}$
\end{tabular}




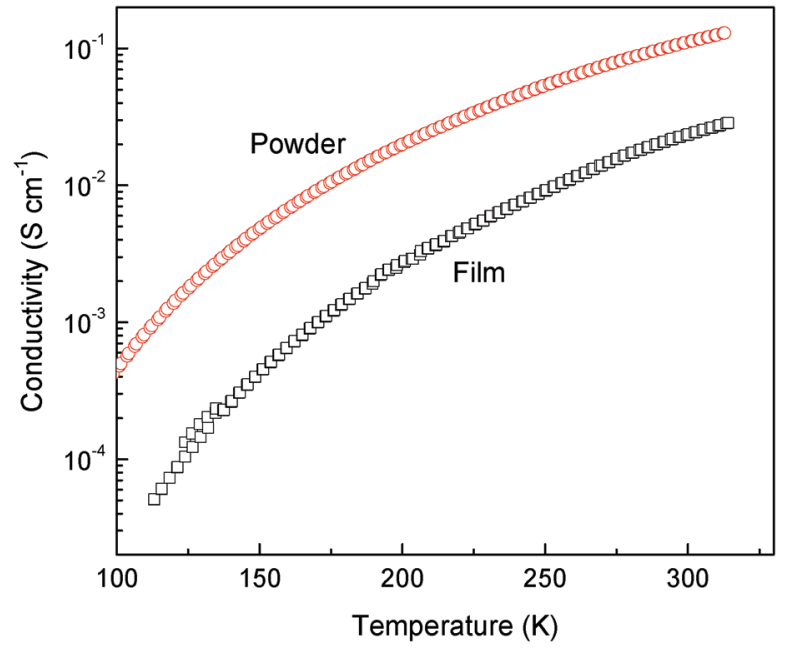

Fig. 6 Temperature dependence of the copolymer prepared at $20 \mathrm{~mol} \%$ $p$-PDA in feed in pelletized powder and film cast from the corresponding colloidal dispersion.

The film conductivity of copolymer colloids prepared with $20 \mathrm{~mol} \% \mathrm{p}$-PDA increased from the order of $10^{-5}$ to $10^{-2} \mathrm{~S} \mathrm{~cm}^{-1}$ in the temperature range of $110 \mathrm{~K}$ to $313 \mathrm{~K}$. Due to the presence of insulating PVP, the film conductivity of the copolymer colloids is below the conductivity of the corresponding powder throughout the whole temperature range. Nevertheless, since the colloidal particles with an increased aspect ratio are capable of forming effective conducting pathways, ${ }^{42}$ and the conducting component mass ratio in the present system ( $c a .1 \mathrm{~g}$ copolymer per $1 \mathrm{~g}$ PVP) is much higher than the common percolation threshold of the PANI-colloidal film, ${ }^{43,44}$ it can be said that electron transport in the copolymer-colloid film may mainly take place through the conducting network composed of copolymer nanofibers. So, the film conductivity of the copolymer colloid prepared with $20 \mathrm{~mol} \% p$-PDA showed similar conductometric sensitivity to temperature as its powder form counterpart. The ratio of the film conductivity at $300 \mathrm{~K}$ to that at $100 \mathrm{~K}$ is over 900 , which is even higher than that of the powder form, 235. Obviously, the poly(aniline-co-p-PDA) in the colloidal dispersion form, which possesses excellent processability and a steep temperature dependence of film conductivity, will be attractive for the development of flexible temperature sensor devices.

\section{Conclusions}

The conductivity of copolymers of aniline with $p$-phenylenediamine decreases with increasing content of the latter comonomer. The temperature dependence of conductivity becomes at the same time steeper, i.e. the electrical properties of copolymers are more sensitive to temperature changes. This may be beneficial in the design of temperature-sensitive sensors, such as pellistors. The colloidal forms of copolymers are composed of nanofibres. Because of the extended morphology of copolymer particles, the films cast from colloidal dispersions maintain a good level of conductivity despite the presence of a non-conducting polymer stabilizer. Due to effective conducting pathways formed by copolymer colloidal particles with a significantly increased aspect ratio, the film conductivity still retains a steep temperature dependence over a wide temperature range. Aqueous colloidal dispersions are stable, easy to be scaled up, and they are suitable for the processing of conducting copolymers by printing techniques.

\section{Acknowledgements}

The authors thank the Czech Science Foundation (14-05568P) for financial support. Y. L. participated in the Postgraduate Course in Polymer Science 2015/6 organized by the Institute of Macromolecular Chemistry in Prague. Dr J. Prokeš from the Charles University in Prague is gratefully acknowledged for providing the data depicted in Fig. 2.

\section{References}

1 J. Stejskal, Prog. Polym. Sci., 2015, 41, 1-31.

2 B. Lakard, G. Herlem, S. Lakard and B. Fahys, THEOCHEM, 2003, 638, 177-187.

3 T. X. Li, C. Q. Yuan, Y. H. Zhao, Q. L. Chen, M. Wei and Y. M. Wang, High Perform. Polym., 2012, 25, 348-353.

4 J. Prokeš, I. Křivka, R. Kužel, J. Stejskal and P. Kratochvíl, Int. J. Electron., 1996, 81, 407-417.

5 H. Q. Tang, A. Kitani, S. Maitani, H. Munemuran and M. Shiotani, Electrochim. Acta, 1995, 40, 849-857.

6 X. Wang and P. Liu, J. Ind. Eng. Chem., 2014, 20, 1324-1331.

7 O. Starykov, J. Prokeš and I. Křivka, Mol. Cryst. Liq. Cryst., 2002, 385, 33-42.

8 R. Holze, Electrochim. Acta, 2011, 56, 10479-10492.

9 S. Karthikeyan, H. M. Pandya, M. U. Sharma and K. Gopal, J. Environ. Nanotechnol., 2015, 4, 1-14.

10 M. A. Shenashen, M. M. Ayad, N. Salahuddin and M. A. Youssif, React. Funct. Polym., 2010, 70, 843-848.

11 P. Bober, J. Stejskal, M. Trchová, J. Prokeš and I. Sapurina, Macromolecules, 2010, 43, 10406-10413.

12 Z. D. Zujovic, Y. Wang, G. A. Bowmaker and R. B. Kaner, Macromolecules, 2011, 44, 2735-2742.

13 Y. Li, G. C. Li, H. G. Peng, Y. Qin and K. Z. Chen, Synth. Met., 2013, 164, 42-46.

14 M. A. Smirnov, M. P. Sokolova, N. V. Bobrova, I. A. Kasatkin, E. Lahderanta and G. K. Elyashevich, J. Power Sources, 2016, 304, 102-110.

15 L. Chao, K. S. Ho, S. Y. Shen, H. Y. Pu, T. H. Hsieh, C. W. Kuo and B. H. Tseng, J. Appl. Polym. Sci., 2013, 127, 1853-1862.

16 C. H. Yang, J. Electrochem. Soc., 1997, 144, 2078-2084.

17 M. H. Lee, Y. C. Luo and J. S. Do, J. Power Sources, 2005, 146, 340-344.

18 P. Fu, H. Li, J. Sun and Z. G. C. Yi, Prog. Org. Coat., 2013, 76, 589-595.

19 X. Rong, F. Q. Zhao and B. Z. Zeng, Talanta, 2012, 98, 265-271. 
20 M. Rani, R. Ramachandran and S. Kabilan, Synth. Met., 2010, 160, 678-684.

21 B. Yang, Micro Nano Lett., 2014, 9, 149-152.

22 T. Siva, K. Kamaraj and S. Sathiyanarayanan, Prog. Org. Coat., 2014, 77, 1807-1815.

23 J. Trlica, P. Sáha, O. Quadrat and J. Stejskal, Eur. Polym. J., 2000, 36, 2313-2319.

24 A. Parsa, M. Sadeghi, Z. Parsa, A. Shakeri, M. Tehrani and S. Ab Ghani, J. Iran. Chem. Soc., 2015, 12, 889-895.

25 A. S. Al-Hussaini and W. Eldars, Des. Monomers Polym., 2014, 17, 458-465.

26 Y. Haldorai, W. S. Lyoo and J. J. Shim, Colloid Polym. Sci., 2009, 287, 1273-1280.

27 J. Stejskal, J. Polym. Mater., 2001, 18, 225-228.

28 B. Weng, R. L. Shepherd, K. Crowley, A. J. Killard and G. G. Wallace, Analyst, 2010, 13, 2779-2789.

29 M. Taunk and S. Chand, Mater. Sci. Semicond. Process., 2015, 39, 659-664.

30 P. Chutia and A. Kumar, Polym. Eng. Sci., 2015, 5, 995-1002.

31 J. Prokeš, J. Stejskal, I. Křivka and E. Tobolková, Synth. Met., 1999, 102, 1205-1206.

32 A. J. Heeger, N. S. Sariciftci and E. B. Namdas, Semiconducting and Metallic Polymers, University Press, Oxford, 2010.
33 M. Trchová and J. Stejskal, Pure Appl. Chem., 2011, 83, 1803-1817.

34 R. H. Sestrem, D. C. Ferreira, R. Landers, M. L. A. Temperini and G. M. do Nascimento, Polymer, 2009, 50, 6043-6048.

35 F. Cataldo, Eur. Polym. J., 1996, 32, 43-50.

36 P. Magdziarz, P. Bober, M. Trchová, Z. Morávková, M. Bláha, J. Prokeš and J. Stejskal, Polym. Int., 2015, 64, 496-504.

37 J. Stejskal, I. Sapurina and M. Trchová, Prog. Polym. Sci., 2010, 35, 1420-1481.

38 H. D. Tran, Y. Wang, J. M. D'Arcy and R. B. Kaner, ACS Nano, 2008, 2, 1841-1848.

39 X. P. Sun and M. Hagner, Langmuir, 2007, 23, 10441-10444.

40 G. R. Zhang, A. J. Zhang, X. L. Liu, S. F. Zhao, J. B. Zhang and J. X. Lu, J. Appl. Polym. Sci., 2010, 115, 2635-2647.

41 N. R. Chiou and A. J. Epstein, Adv. Mater., 2005, 17, 1679-1683.

42 Y. Li, P. Bober, D. H. Apaydin, T. Syrový, N. S. Sariciftci, J. Hromádková, I. Sapurina, M. Trchová and J. Stejskal, Synth. Met., 2016, 221, 67-74.

43 E. C. Cooper and B. Vincent, J. Phys. D: Appl. Phys., 1989, 22, 1580-1585.

44 X. G. Li, M. R. Huang, J. F. Zeng and M. F. Zhu, Colloids Surf., A, 2004, 248, 111-120. 